\title{
Machine Intelligence
}

\author{
Matthew N. O. Sadiku' ${ }^{1}$, Damilola S. Adesina ${ }^{2}$, Sarhan M. Musa ${ }^{3}$ \\ Roy G. Perry College of Engineering, Prairie View A\&M University, Prairie View, TX 774466,2,3
}

\begin{abstract}
Researchers worldwide have always attempted to put human intelligence into machines by the ways of algorithms. Machine intelligent results from the development of Artificial Intelligence (AI). The goal of machine intelligence is giving machines the ability to learn from the changing environment and making predictions. The paper provides a brief introduction to machine intelligence.
\end{abstract}

Keywords: Machine Intelligence, Intelligent Machines, Artificial Intelligence

\section{INTRODUCTION}

The traditional machine was a physical system that lacked intelligence. It was just a simple machine that was intended to substitute human's physical labor [1]. The idea of creating thinking, reasoning machines has been appealing and fascinating to human imagination. Several human activities (such as engaging in conversation or driving a car) requires intelligence.

All our activities as human beings can be described by five components: data, prediction, judgment, action, and outcomes. Machine intelligence (MI) lowers the cost of goods and services that rely on prediction. This is important since prediction is an input to a host of activities including transportation, agriculture, healthcare, energy manufacturing, and retail [2].

\section{WHAT IS INTELLIENCE?}

There is no exact and universally accepted definition of intelligence in humans or machines. An intelligent machine that can perform tasks would be helpful in almost any human endeavor. Larry Fogel identified three key elements of intelligence [3]: (1) ability to predict, (2) ability to adapt, and (3) ability to take appropriate action.

Machine intelligence is basically a prediction technology. Since prediction can have a profound effect on our life, we often make efforts to predict our future from the past and the present. Adaptability is a vital component of any intelligent machine; it has already been introduced in several automatic devices.

We can also consider intelligence as consisting of mainly communication and learning [4]:

1. Ability to communicate: It is natural to expect an intelligent machine to be able to communicate in human language. Human knowledge and thinking are always mediated using natural language. The intelligent machine can be a computer that is programmable. It must be able to communicate in an interactive manner.

2. Ability to learn: An intelligent machine must be able to learn. Learning enables the machine to adapt and modify itself based on experience and external environment. There are several types of behavior that can be regarded as learning. Machine learning can be related to control theory and artificial intelligence. Today machine learning requires large amounts of data.

\section{APPLICATIONS}

There are now machines which show adaption and learning; machines which can classify speech and hand-writing; machines which can perform goal directed motor activity; machines which play games; and machines which can communicate [5]. Here are just a few applications of MI.

- Robots: Robotics is undergoing explosive growth due to improvements in computational power, new materials, and better sensors. For example, car manufacturers use automation in form of welding and assembly robots. 
International Journal of Advanced Research in Computer and Communication Engineering

Vol. 9, Issue 2, February 2020

- Games: Machine intelligence is used in games. The Checkers program CHINOOK was the first official man-machine world champion in any game in 1994. Hex is another challenging zero-sum board game that involves two players [6]. Watson was developed by IBM as a question answering computer system capable of answering questions posed in natural language.

- Space Exploration: Through telecommunication technology, humans can control space vehicles at remote places. The concept of teleoperation is important in space program. NASA has identified five directions for developing intelligent machines [7]: (1) automated operations in space, (2) precision pointing and control, (3) efficient data acquisition, (4) real-time data management, and (5) low-cost data distribution.

\section{CHALLENGES}

In today's world, human intelligence is still far superior to machine except in the ability to process, compute, store, and distribute data at fast rates. For example, machine intelligence never independently produced an original idea or discovered any new principle. An intelligent machine has never come up with a solution to a social problem or a crime [8].

Some perceive that although intelligent machines can serve as useful tools, they can also be used for dubious purposes. The same can be said of any human invention such as computers, vehicles, guns, robots, etc. [9].

Since the beginning of the Industrial Revolution, workers have been uprooted progressively by new machines. However, the new machines cannot replace the engineers who invented, designed, and produced them [10].

Measuring "machine intelligence" is hard. There is no absolute measure; only relative. Some have introduced Machine Intelligence Quotient (MIQ) as a new index to represent machine intelligence [11].

\section{CONCLUSION}

Learning and applying knowledge is a crucial to human intelligence. Collective intelligence that builds on the human intelligence is different from Turing machine intelligence. The intelligent machines should have the ability to learn, adapt, and predict [12]. Machine prediction is cheaper and better substitute for human prediction, just as machines outperform humans in arithmetic. As MI lowers the cost of prediction, we will start using it as an input for things for which we never previously did.

More information about machine intelligence can be obtained from books on Amazon.com and the journal exclusively devoted to it: Nature Machine Intelligence.

\section{REFERENCES}

[1]. S. Wang and H. Di, "Machine Intelligence and Intelligent Machines," Proceedings of 2011 International Conference on Fluid Power and Mechatronics, 2011, pp. 487-491.

[2]. A. Agrawal, J. Gans, and A. Goldfarb, "The simple economics of machine intelligence," November 2016, https://hbr.org/2016/11/the-simpleeconomics-of-machine-intelligence

[3]. Z. Michalewicz and M. Michalewicz, "Machine intelligence, adaptive business intelligence, and natural intelligence," IEEE Computational Intelligence Magazine, February 2008, pp. 54-63.

[4]. T. Mikolov, A. Joulin, and M. Baroni, "A roadmap towards machine intelligence," https://arxiv.org/pdf/1511.08130.pdf

[5]. N. L. Collins and D. Michie (eds), Machine Intelligence. Edinburgh, UK: Oliver \& Boyd. 1967.

[6]. S. K. Chalup, D. Mellor, and F. Rosamond, "The Machine Intelligence Hex Project," Computer Science and Education, Volume: 15, No. 4, 2005, pp. 245-273.

[7]. Machine Intelligence and Robotics: Report of the NASA Study Group, March 1980, http://www.rr.cs.cmu.edu/NASA\%20Sagan\%20Report.pdf

[8]. M. Lee, "10 Questions for Machine Intelligence," The Futurist, Septemer-October 2014, p. 64.

[9]. D. Beyer, The Future of Machine Intelligence. Sebastopol, CA: O'Reilly Media, 2016.

[10]. K. A. Toth, "The Workless Society: How Machine Intelligence Will Bring E," The Futurist, vol. 24, no.3, May/Jun 1990, pp. 32-37.

[11]. H. J. Park, B. K. Kim, and G. Y. Lim, "Measuring machine intelligence for human-machine cooperative systems using intelligence task graph," Proceedings of the 1999 International Conference on Intelligent Robots and Systems, 1999, pp. 689-694.

[12]. J. Ma, S. Chen, and Y. Xu, "Fuzzy logic from the viewpoint of machine intelligence," Fuzzy Sets and Systems, vol. 157, 2006, pp. 628-634. 


\section{IJARCCE}

International Journal of Advanced Research in Computer and Communication Engineering

Vol. 9, Issue 2, February 2020

\section{BIOGRAPHIES}

Matthew N.O. Sadiku is a professor in the Department of Electrical and Computer Engineering at Prairie View A\&M University, Prairie View, Texas. He is the author of several books and papers. His areas of research interest include computational electromagnetics and computer networks. He is a fellow of IEEE.

Damilola S. Adesina is a doctoral student at Prairie View A\&M University. His research work is currently focused on optimizing communication systems using machine learning and deep learning.

Sarhan M. Musa is a professor in the Department of Engineering Technology at Prairie View A\&M University, Texas. He has been the director of Prairie View Networking Academy, Texas, since 2004. He is an LTD Sprint and Boeing Welliver Fellow. 